\title{
Prolonged Prenatal Psychotropic Medication Exposure Alters Neonatal Acute Pain Response
}

\author{
TIM F. OBERLANDER, RUTH ECKSTEIN GRUNAU, COLLEEN FITZGERALD, \\ ANN-LOUISE ELLWOOD, SHAILA MISRI, DAN RURAK, AND KENNETH WAYNE RIGGS
}

Department of Pediatrics [T.F.O., R.E.G., C.F.], Reproductive Mental Health, Department of Psychiatry [S.M.], and Pharmaceutical Sciences [W.R.], University of British Columbia, Vancouver, BC, Canada; Biobehavioral Research Unit, Centre for Community Child Health Research [T.F.O., R.E.G., C.F.], Fetal Maternal Medicine [D.R.], BC Institute for Children's and Women's Health, Vancouver, BC, Canada; Department of Psychology, University of Calgary, Calgary, AB, Canada [A.-L.E.]

\begin{abstract}
Selective serotonin reuptake inhibitors (SSRIs) and benzodiazepines are frequently used to treat maternal depression during pregnancy, however the effect of increased serotonin (5HT) and $\gamma$-amino-butyric acid (GABA) agonists in the fetal human brain remains unknown. 5HT and GABA are active during fetal neurologic growth and play early roles in pain modulation, therefore, if prolonged prenatal exposure alters neurodevelopment this may become evident in altered neonatal pain responses. To examine biologic and behavioral effects of prenatal exposure, neonatal responses to acute pain (phenylketonuria heel lance) in infants with prolonged prenatal exposure were examined. Facial action (Neonatal Facial Coding System) and cardiac autonomic reactivity derived from the relationship between respiratory activity and short term variations of heart rate (HRV) were compared between 22 infants with SSRI exposure (SE) [fluoxetine $(n=7)$, paroxetine $(n=11)$, sertraline $(n=4)] ; 16$ infants exposed to SSRIs and clonazepam (SE +$)$ [paroxetine $(n=14)$, fluoxetine $(n$ $=2)$ ]; and 23 nonexposed infants during baseline, lance, and recovery periods of a heel lance. Length of maternal SSRI use did not vary significantly between exposure groups-[mean (range)] SE:SE + 183 (31-281):141 (54-282) d ( $p>0.05)$. Infants exposed to SE and SE+ displayed significantly less facial activity to heel lance than control infants. Mean HR increased with lance, but was significantly lower in SE infants during recovery. Using measures of $\mathrm{HRV}$ and the transfer relationship between heart rate and respiration, SSRI infants had a greater return of parasympathetic cardiac modulation in the recovery
\end{abstract}

\section{ABSTRACT}

period, whereas a sustained sympathetic response continued in the control group. Prolonged prenatal SSRI exposure appears to be associated with reduced behavioral pain responses and increased parasympathetic cardiac modulation in recovery following an acute neonatal noxious event. Possible 5HT-mediated pain inhibition, pharmacologic factors and the developmental course remain to be studied. (Pediatr Res 51: 443-453, 2002)

\author{
Abbreviations \\ 5HT, 5 hydroxytryptamine (serotonin) \\ BZ, benzodiazepine \\ C, control (nonexposed) infants \\ DA, dopamine \\ GABA, $\gamma$-amino-butyric acid \\ HFP, high-frequency power \\ HRV, heart rate variability \\ LFP, low-frequency power \\ NE, norepinephrine \\ NFCS, Neonatal Facial Coding System \\ NICU, neonatal intensive care unit \\ PKU, phenylketonuria \\ $\mathbf{R P}$, respiratory power \\ RSA, respiratory sinus arrhythmia \\ SE, SSRI-exposed infants \\ SE+, SSRI- and BZ-exposed infants
}

SSRI, selective serotonin reuptake inhibitors
Maternal anxiety and depression are frequently treated during pregnancy with psychotropic medications such as SSRIs and BZs (1). Given that all psychotropic medications diffuse easily across the placenta, clinicians caring for women with

Received January 31, 2001; accepted October 16, 2001.

Correspondence and reprint requests: Tim F. Oberlander, MD, FRCPC, Biobehavioral Research Unit, Centre for Community Health and Health Evaluation Research, Room L408, 4480 Oak Street, Vancouver, BC V6H 3V4, Canada; e-mail: toberlander@cw.bc.ca

This work was supported by funding from the British Columbia Medical Services Foundation. psychiatric illness during their pregnancies are faced with the difficult task of making recommendations regarding psychotropic medication use with unknown effects on the growing fetus. To date, none of these medications have been approved for use during pregnancy by the Food and Drug Administration (1), however, the widespread use of SSRIs alone or in combination with BZs to treat anxiety and depression during pregnancy has raised many unanswered questions about the longterm neurodevelopmental effects following prenatal exposure. 
At pharmacologically active doses, SSRIs inhibit the reuptake of 5HT at the presynaptic junction, leading to increased concentrations of this neurotransmitter in the synaptic cleft. Throughout the human neuroaxis, GABA acts as an inhibitory amino acid transmitter that produces membrane hyperpolarization through a variety of GABA receptors. GABA agonist medications, such as BZs exert their pharmacologic effects via binding to specific sites on the GABAa receptor.

Before assuming their roles as neurotransmitters, both 5HT and GABA appear early in embryogenesis as developmental signals involved in regulating the morphogenesis of both GABA and monoamine (i.e. 5HT, NE, and DA) systems $(2,3)$, such as cell migration, axonal outgrowth, and synaptogenesis, coordinating mediators of intercellular communication $(4,5)$, and modulation of respiratory rhythm (6) in the developing fetus, (7-10). The trophic action of GABA, mediated by GABAa receptors, on developing neurons (see Ref. 10 for review) appears to involve the regulation of $\mathrm{Ca}^{2+}$ homeostasis and interestingly it acts as a trophic factor for monoamine (i.e. 5HT) neuronal function (7-9). The functional implications of disrupting these processes are not well understood.

In the growing fetal and infant brain, both 5HT and its co-neurotransmitter GABA have important inhibitory roles regulating pain signals (11-13). Monoamines (5HT) play key roles in the modulation of nociception at all levels of the central nervous system (CNS) extending from cutaneous surfaces, the dorsal horn of the spinal cord to the thalamus, and higher limbic cortical structures responsible for conscious appreciation of pain. Similarly, the GABAergic system is one of the earliest neurotransmitter systems to develop. GABA receptor agonists, such as BZs, facilitate the effects of GABA at the GABAa receptor. High concentrations of GABA and GABA receptors have been found in regions of the CNS that are implicated in the afferent transmission of nociceptive information or the production of antinociception (spinal cord, medullary and pontine nuclei, and thalamus.) (14).

With increased levels of 5HT after SSRI exposure, cortical morphology in monoaminergic-rich regions of the brain and general monoaminergic ontogeny and function may be altered. In animal models, prenatal exposure to SSRI medication produces a variety of adverse neuroanatomic and behavioral consequences, though outcomes are not consistent $(4,15)$ and the functional consequences are uncertain. Prenatal exposure to these antidepressants has been shown to result in both reduced numbers as well as function of monoaminergic receptors (16) in neonatal in rats (17); findings that persist in the mature animal (18). Comparable human data are lacking. Fluoxetine is regarded as safe to use during pregnancy (1) with little or no risk of congenital malformations (19-22). However, little is known about the neurobehavioral function in infants following in utero exposure to SSRIs (23-25). Case reports have described infants with pre- and postnatal fluoxetine exposure and marked acrocyanosis, tachypnea, temperature instability, irritability, and elevated drug levels (26-28). Cohen (29) reported that among a cohort of 64 infants with late gestation fluoxetine exposure, there was a higher frequency of poor neonatal adaptation, jitteriness, tachypnea, hypoglycemia, hypothermia, poor tone, and a weak or absent cry. Similarly, in a prospective study of 228 pregnant women taking fluoxetine, Chambers (30) reported that infants with late gestation exposure had higher rates of premature delivery, admission to special-care nurseries, and poor neonatal adaptation. Birth weight was also lower and birth length shorter in infants exposed to fluoxetine late in gestation. Chambers also observed fluoxetine-exposed infants had a higher risk for three or more minor anomalies, even after $\mathrm{BZ}$ exposure was controlled for, suggesting that the effect of prenatal exposure might have subtle effects on the developing brain that only become apparent later in infancy. Beyond these reports, the duration of these symptoms beyond the immediate postnatal period and relationship to infant development is unknown.

Given the dual roles of GABA and 5HT as trophic agents and as neurotransmitters, it is conceivable that alterations to the prenatal levels of these neurochemicals and the developmental ontogeny of these systems may lead to changes in infant pain behaviors. In this context, pain response in the newborn period may be a sensitive probe of altered neurodevelopment after prolonged prenatal exposure. Further, because 5HT and GABA are active in modulation (i.e. inhibition) of pain signals, we hypothesized that prolonged prenatal exposure and hence increased levels of inhibitory neurotransmitters at developmental sensitive periods would blunt a typical neonatal pain response. In the current study, biobehavioral responses to an acute noxious event (PKU heel lance) on the second day life were examined in infants with prolonged prenatal psychotropic medication exposure and compared with nonexposed control term born infants.

\section{METHODS}

\section{Subjects}

With approval from the University of British Columbia Research Ethics Board, Children's and Women's Health Center of British Columbia Research Review Committee, and informed parent consent, a consecutive cohort comprised of mothers and their infants were recruited during pregnancy as a part of a larger study of psychotropic medication use pre- and postpartum. Two groups of medication exposed infants were studied while undergoing heel lance PKU blood collection (Table 1); 22 with prenatal SSRI exposure (SE) [paroxetine ( $n$ $=11)$, fluoxetine $(n=7)$, sertraline $(n=4)]$; 16 infants with SRRI [paroxetine $(n=14)$, fluoxetine $(n=2)$ ] combined with the benzodiazepine clonazepam ( $\mathrm{SE}+$ ), and 23 control term born nonexposed infants (C). A control group of term born healthy infants and mother dyads were recruited at birth and studied during the PKU blood collection for comparative purposes. Of the 82 eligible mothers approached during pregnancy while on medication, 52 were recruited, and the remainder declined. Fourteen infants in the medication group were not included for analysis due to technical reasons. Of the 26 control infants studied, 3 were eliminated from the final data set for technical reasons. All infants underwent the blood collection without complications. Criteria for inclusion were birth weight $2500-3200 \mathrm{~g}$ and/or $\geq 37$ wk gestational age at birth, and absence of congenital heart or CNS lesions. Median 
Table 1. Infant characteristics at birth

\begin{tabular}{lccr}
\hline & & Group (no.) & \multicolumn{1}{c}{$\mathrm{C}(23)$} \\
\cline { 2 - 4 } & SE* $(22)$ & SE+†(16) & $39.2(37.0-41.6)$ \\
Birth age (wk) & $39.5(37.3-42.0)$ & $39.6(37.6-41.6)$ & $3485(2690-4150)$ \\
Birth wt (g) & $3401(2703-4270)$ & $3490(2865-4240)$ & $35.0(38.5-32)$ \\
Head circumference & $34.3(32-37)$ & 34.532 .5036 .00 & $51.6(47-57)$ \\
Length & $51.84(47-64)$ & $51.5(49-54.5)$ & $8.00(1-9)$ \\
Apgar score 1 min (median) & $7.00(3-9)$ & $8.00(3-9)$ & $9.00(8-9)$ \\
Apgars score 5 min (median) & $9.00(7-9)$ & $9.00(6-9)$ & \\
\hline
\end{tabular}

* Fluoxetine $(n=7)$, paroxetine $(n=11)$, sertraline $(n=4)$.

$\dagger$ Fluoxetine $(n=2)$, paroxetine $(n=14)$ with clonazepam.

doses of SSRIs, BZ, and the length of exposure did not differ between groups.

\section{Procedure}

The study was conducted in a specially designated room adjacent to the newborn nursery in Children's and Women's Health Center of British Columbia while infants underwent the routine heel lance blood collection screening for metabolic diseases (PKU). A research nurse recruited all infants. The infant was seated on his/her mother's lap during the procedure. Recording began when the infant was in an awake, alert, noncry state (31).

Behavioral data acquisition. A camera technician carried out video-recording during baseline, lance, and recovery periods. The NFCS $(32,33)$ was used to provide detailed facial activity. NFCS-trained coders, blind to the subject group and all information about the infants, coded the presence or absence of a discrete set of precisely defined facial actions, using slow motion and stop frame playback technique. From the NFCS, seven facial actions were coded (brow bulge, eye squeeze, nasolabial furrow, open mouth, vertical mouth stretch, horizontal mouth stretch, taut tongue), as they have been associated with reactivity to nociceptive procedures in full term healthy neonates $(33)$, premature neonates $(34,35)$, and in infants up to age 18 mo of age (36).

Facial action from the last $20 \mathrm{~s}$ of baseline, and first $20 \mathrm{~s}$ from the lance and $20 \mathrm{~s}$ from the recovery were coded in random order. One coder carried out the NFCS coding, after reaching at least 0.85 interrater reliability during training. Reliability was calculated according to the conservative formula described elsewhere (32) that assess the proportion of agreement on actions recorded by two coders relative to the total number of actions coded as occurring.

Physiologic signal acquisition. Analysis of short-term variations in heart rate that follows a noxious event were used to examine the neurologic sequelae following prenatal psychotropic medication exposure. Variability in HR is mediated primarily by changing levels of parasympathetic and sympathetic outflow from the CNS to the sinoatrial node at the heart. The HR power spectrum typically contains a peak at the respiratory frequency representing RSA and peaks at lower frequencies between 0.01 and $0.15 \mathrm{~Hz}$. Studies using selective pharmacologic blockade of the cardiac sympathetic and parasympathetic receptors have shown that fluctuations in heart rate above $0.15 \mathrm{~Hz}$ are mediated exclusively by changes in para- sympathetic activity, whereas lower frequency changes are mediated jointly by changes in parasympathetic and sympathetic efferent activity (37). Thus, with spectral analysis, shortterm HR variability can be used to quantify changing levels of cardiac autonomic modulation.

Parasympathetic influences affecting HR via the vagus nerve are easier to identify, therefore these autonomic influences have been studied more than the diffuse sympathetic influences. Most of the work investigating autonomic modulation of HR has focused on variations in HR that occur with respiration (i.e. $0.15-1.0 \mathrm{~Hz}, \mathrm{RSA}$ ) and has lead to the suggestion that infant HR variability associated with RSA may be a measure of individual differences in stress response, and the functional status of the CNS and autonomic control mechanisms $(38,39)$. This vagal tone, however, may not quantify the entire spectrum of autonomic arousal and this limitation has raised concern about its broad application as a biopsychologic probe (40). Moreover, the magnitude of RSA is influenced by both respiratory rate and tidal volume (i.e. RSA increases as respiratory rate slows and tidal volume increases). Thus, the magnitude of the RSA may be a measure of cardiac vagal activity only when respiratory activity remains stable, which is rarely the case in a clinical setting with infants - particularly during exposure to noxious stimuli.

To account for the empirical limitations of spectral analysis of HRV, the mathematical technique, transfer function analysis (TFA), was used to derive specific measures of parasympathetic and sympathetic modulation of HR from the relationship between short-term changes in HR (RSA) and respiratory activity $(37,41,42)$. Use of transfer function analysis of HRV and respiratory activity has been previously used to demonstrate a maturational increase in sympathetic modulation of heart rate during quiet sleep in healthy preterm infants (43) and the autonomic effects of noxious events and anesthesia in term and former preterm infants (41-44).

Three standard surface chest electrodes were used to produce continuous ECG, and a two-belt microprocessorcontrolled inductance plethysmograph system was used to produce a respiratory signal (Respiratrace-Plus, NonInvasive Monitoring Systems, Miami, FL, U.S.A.). Both signals were digitally sampled to disk at $360 \mathrm{~Hz}$ using a personal computerbased data acquisition system (HR View Software, Boston Medical Technologies, Boston, MA, U.S.A.). Calibration of the respiratory signal was performed using a previously described algorithm (45), assuming an infant tidal volume of 7 
$\mathrm{mL} / \mathrm{kg}$. Respiratory volumes were normalized to a standard body surface area of $1.73 \mathrm{~m}^{2}$ to enable comparisons between previous adult and infant respiratory data $(41,42)$. One lead of surface ECG and respiratory activity were recorded continuously during baseline, lance, and recovery periods.

$\mathrm{R}$ waves were detected from the sampled ECG and used to form a smoothed instantaneous $4 \mathrm{~Hz} \mathrm{HR}$ time series (46). The inductance respiratory signal (RP) was digitally low-passfiltered and decimated to $4 \mathrm{~Hz}$. Segments of HR and respiratory activity $(2.2 \mathrm{~min}$ each) were selected from 1) the resting baseline period within $5 \mathrm{~min}$ before the lance, 2) a lance period starting within $20 \mathrm{~s}$ after the heel prick blood collection, and 3) a recovery period within $7 \mathrm{~min}$ after the lance. The epoch selection criteria were based on quantitative assessment of signal stability and the absence of gross movement artifact according to previously reported procedures $(41,42)$.

Power spectral estimates of HR were quantified using the area (power) of the spectrum in a low-frequency region (LF: $0.04-0.15 \mathrm{~Hz}$ ) and a high-frequency region (HF: $0.15-0.80$ $\mathrm{Hz}$ ), as well as by the ratio of LFP and HFP power (LFP/HFP), as previously described (37). Similar measures of respiratory activity were tabulated from the respiratory power spectrum to yield total respiratory power (RP) (LF-RP and HF-RP).

To further determine the contribution of both sympathetic and parasympathetic components to HR modulation, the effects of respiratory activity on heart rate was assessed using transfer function analysis, as previously reported in infants $(41,42)$ and adults (37). Autospectra of the HR and respiratory signals and the cross spectrum between them were estimated for each 128 -s (512 points) segment as previously described (47). The complex transfer function, or frequency response, between respiratory activity and HR was quantified using the crossspectral method to yield magnitude (gain) and phase components. A squared coherence spectrum was also computed to define the degree of the linear relation between respiratory activity and HR. The coherence varied between 0 and 1 .

Quantitative measures of cardiac parasympathetic and sympathetic cardiac control were derived from the average coherence weighted transfer gains of the 2.2-min segments of data as previously reported in infants $(42,44)$. Using this technique, previous work during pharmacologic treatment of adults with either atropine while upright, or propranolol while supine, demonstrated transfer gain and phase plots characteristic of pure parasympathetic and pure sympathetic modulation of HR (37), respectively. A pure sympathetic HR response (during standing with atropine) was characterized by a reduced gain at frequencies $>0.01 \mathrm{~Hz}$ and a phase delay. In contrast, under pure vagal conditions (supine plus propranolol), the heart rate response was characterized by higher gain at all frequencies and no phase delay. This technique has been previously used to study pain reactivity and responses to anesthetics in infants $(41,42,44)$.

\section{Statistical Analysis}

A group by time repeated measure ANOVA was used to compare outcome measures across study periods. Post hoc comparisons were conducted where appropriate. A difference was considered statistically significant for $p$ values $<0.05$. To determine the significance of differences of change from baseline for cardiac autonomic measures, analysis of covariance was used to examine the effect initial or starting measure had on lance response. To examine differences in facial activity between the groups across time periods during the lance event, a one-way ANOVA was used.

Behavioral data. To examine the pattern of facial response over time, occurrence of each of the seven NFCS facial actions were summed within each of 10, 2-s time segments, to yield 10 facial scores with a possible range of $0-7$, for each event. One infant's data were missing for the recovery event, as the face was out of view. To retain equal cell sizes in the ANOVA, the infant's pain score for the event was replaced with the mean value for that cell, i.e. the mean values observed for infants in that group during the recovery event.

Physiologic data. The mean and SEM of the HR, respiratory activity, and power spectra for each data segment were calculated. For the purposes of display, group average transfer function estimates of both gain and phase from each experimental epoch were computed as previously described (37).

\section{RESULTS}

All subjects were born at the end of term pregnancies, without major complications at birth or significant differences in demographic variables between groups (Table 1). None of the infants were admitted to a special care nursery/NICU for care in the newborn period. Exposure to medications occurred in the last two trimesters and the length of maternal SSRI use did not vary significantly between the $\mathrm{SE}$ and $\mathrm{SE}+$ exposure groups (Table 2). With the exception of one, all mothers took their regular medication in the $3 \mathrm{~d}$ before and after birth, and thus we assumed that all maternal medication levels were at a steady state at the time of the study. The majority of infants were breast fed and differences in background characteristics between breast-feeding and non-breast-feeding infants were not significant.

\section{NFCS Facial Pain Response}

Facial activity increased significantly from baseline to lance in all groups, and decreased from the lance to recovery $[F=$ 122; $d f(2,1) ; p<0.01]$. In Figure 1, 10 blocks of 2-s duration are presented in 20-s segments of facial action for each study period. Facial activity did not differ significantly between the groups during baseline or recovery. During lance, reactivity was significantly lower among SE- and SE+-exposed infants, compared with controls, in the first $[F=3.70 ; d f(2,60) ; p=$ $0.03]$ and second $[F=4.44 ; d f(2,60) ; p=0.02]$ time periods of the lance event. After this initial $4 \mathrm{~s}$ of the lance period, the SE group remained lower throughout, but the difference was not statistically significant. Facial action responses did not vary between infant breast-feeding and those not breast-feeding ( $p$ $>0.05)$.

\section{Physiologic Data}

Mean heart rate. Mean HR increased with lance and fell in recovery in all groups. [F=84, $d f(2,1) ; p<0.01]$. However, 
Table 2. Medication exposure characteristics [means (range)]

\begin{tabular}{|c|c|c|c|}
\hline & \multicolumn{3}{|c|}{ Group (no.) } \\
\hline & $\mathrm{SE}^{*}(22)$ & $\mathrm{SE}+\dagger(16)$ & $\mathrm{C}(23)$ \\
\hline Length of exposure SSRI + (mean days) & $183(31-281)$ & $149(54-282)$ & 0 \\
\hline Breast feeding (no.) & 17 & 13 & 20 \\
\hline Age at time of PKU (h) & $51.5(27-117)$ & $53.4(34-129)$ & $50.1(26-121)$ \\
\hline \multicolumn{4}{|l|}{ Maternal analgesia (no.) } \\
\hline Epidural & 11 & 11 & 8 \\
\hline Local & 1 & 0 & 0 \\
\hline \multicolumn{4}{|l|}{$\begin{array}{l}\text { Dose of SSRI at time of delivery } \\
\text { (median, mg/d) (range) }\end{array}$} \\
\hline Paroxetine & $20(10-30)$ & $20(5-40)$ & - \\
\hline Fluoxetine & $20(10-30)$ & $12.5(10-15)$ & - \\
\hline Sertaline & $62.5(50-150)$ & - & - \\
\hline
\end{tabular}

* Fluoxetine $(n=7)$, paroxetine $(n=11)$, sertaline $(n=4)$.

$\dagger$ Fluoxetine $(n=2)$, paroxetine $(n=14)$ with clonazepam.

$\ddagger$ Days before delivery.

mean HR was significantly lower among both SE-exposed infant groups in the recovery period. ( $p=0.04$ ) (Fig. 2). Differences between breast-fed and non-breast-fed infants were not significant $(p>0.05)$.

Power spectral estimates. In both SSRI-exposed groups, LF and HF power decreased significantly $[F=12.7, d f(2,1) ; p<$ 0.01 ; and $F=25.9$, $d f(2,1) ; p>0.01$, respectively] from baseline, and increased again in the recovery period (Fig. 3). LFP differences were not significantly different between groups, however, HFP was significantly higher in the recovery period among both groups of exposed infants. The ratio LFP/ HFP remained stable in both groups across study periods with minimal changes $(F=0.29, p=0.70)$ and no significant differences were observed between groups $(F=0.2, p=0.57)$ (Fig. 3). Finally, total respiratory power increased significantly from baseline with the lance and decreased in the recovery period in both groups $[F=59.1, d f(2,1) ; p<0.01]$ (Fig. 4) with no group differences.

Transfer function estimates of respiratory sinus arrhythmia. Qualitatively, during baseline transfer gain was high at all frequencies in exposed and control groups (Fig. 5, $A$ and $B$ ). Because outcomes were not significant between SE and SE+ groups, for clarity only SE versus control transfer function results are presented in Figure 5. Phase began at $0^{\circ}$ at $0 \mathrm{~Hz}$ and decreased slightly with increasing frequencies, illustrating a predominance of vagal cardiac modulation in the prelance baseline condition. In response to the lance, transfer gain decreased across all frequencies and became very low in the HF range, suggesting increased sympathetic modulation. In contrast to the control group, transfer phase did not fall from baseline among SE-exposed infants, suggesting increased sympathetic cardiac modulation was accompanied by a minimal withdrawal of parasympathetic activity. In the recovery period, group transfer gain among all infants increased across all frequencies while phase remained below $0^{\circ}$, consistent with a return of parasympathetic and sympathetic modulation near baseline levels. However, among SE infants, LF transfer gain increased to levels significantly higher $(p=0.02)$ than controls, reflecting a return to greater levels of parasympathetic modulation.

In summary, from the transfer function results, both exposed and nonexposed infants responded to the heal lance with increased sympathetic and reduced cardiac parasympathetic modulation. However, SE infants responded with maintenance of parasympathetic activity (less parasympathetic withdrawal) with the lance and a markedly increased parasympathetic modulation during recovery than control infants. This was particularly evident among the SE alone group. These results are consistent with the lower mean HR and increased HF spectral power in the recovery phase.

\section{DISCUSSION}

Consistent with our hypothesis, prolonged second and third trimester prenatal psychotropic medication exposure was associated with an attenuated acute pain response in the newborn period. The duration of facial action and cardiac autonomic reactivity were shorter and less intense among SE and SE+ infants compared with nonexposed control infants. In particular, among exposed infants there was a reduced immediate facial response and maintenance (less withdrawal) of parasympathetic cardiac modulation following the lance. Further, during the recovery period, lower mean HR and increased parasympathetic cardiac modulation were present. Importantly, these effects were observed in the absence of the poor perinatal behaviors reported by Chambers (30) and Cohen (29). Although we observed no differences between medicationexposure groups, at this point we are unable to determine to what the extent BZ exposure alone may have influenced pain reactivity.

The mechanism(s) underlying these behavioral outcomes is unclear at present, however, at least two possibilities may 

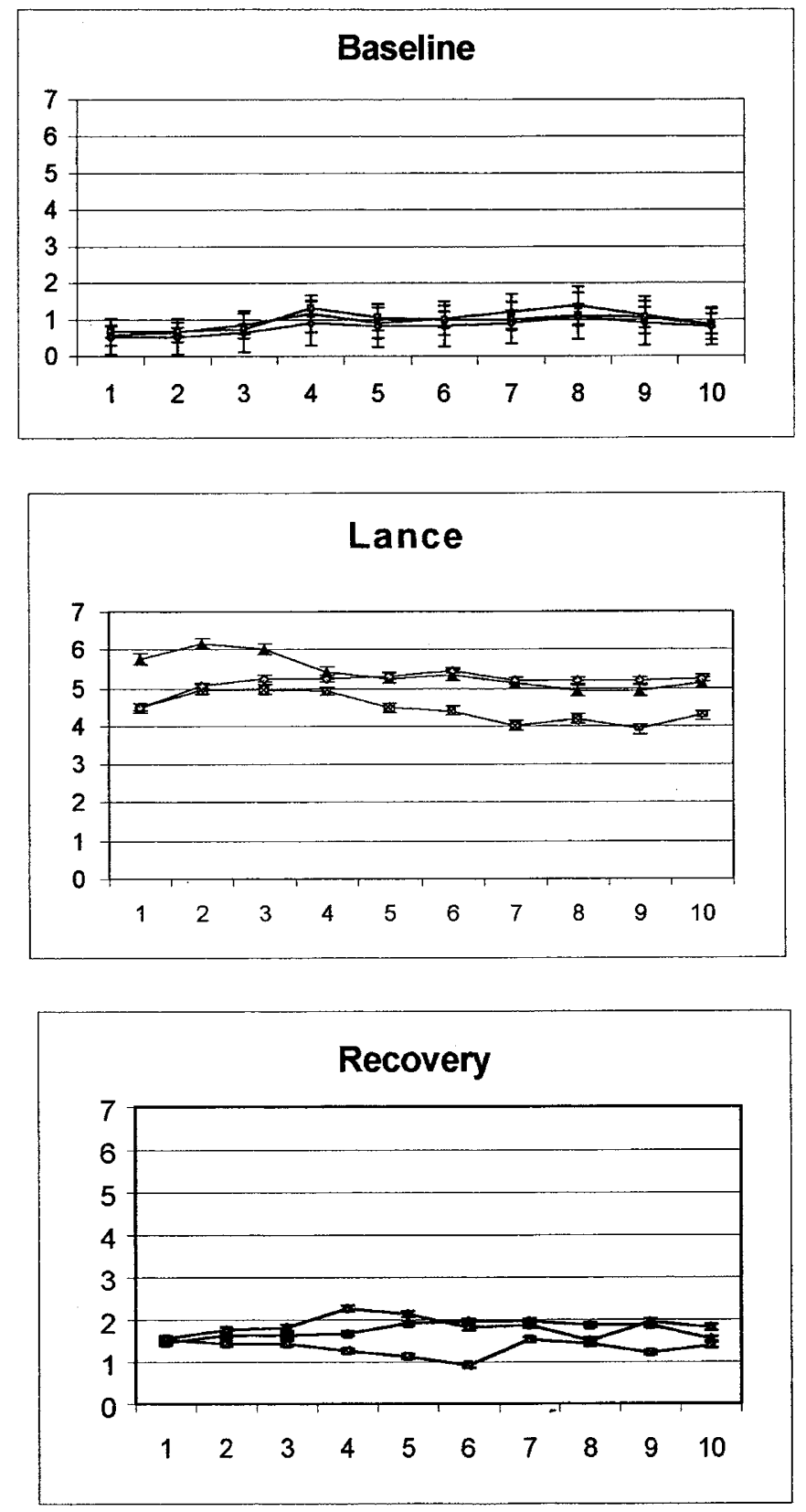

Figure 1. Mean facial pain score across each of ten 2-s events (mean facial pain score \pm SEM per 2-s period). Square, SSRI; triangle, control infants; diamond, SSRI+.

account for our findings. One involves direct pharmacologic effects of the drugs still present in the infants at the time of the PKU test. The other is that we observed the effects of altered brain development due to prolonged in utero exposure to the medications. The pharmacokinetic data collected in a concurrent study from the same cohort indicate the continued presence of all three drugs in infant plasma at this time (48). In the case of fluoxetine, the drug concentrations were the same as in the cord blood of the infants at delivery, whereas for paroxetine and sertraline, the levels at PKU testing were $60 \%$ and $27 \%$, respectively, of the concentrations in cord blood. These differences likely reflect differences in the fetal/neonatal ability to metabolize the compounds. They also resulted in concentra- tions of fluoxetine (mean $=40.7 \mathrm{ng} / \mathrm{mL}$ ) that were substantially higher than the levels of paroxetine $(3.0 \mathrm{ng} / \mathrm{mL})$ and sertraline $(1.6 \mathrm{ng} / \mathrm{mL})$. However, as the latter two agents are more potent inhibitors of brain serotonin uptake compared with fluoxetine (49), the potential pharmacologic effects of the drugs could have been similar. Although all three drugs are excreted in breast milk $(48,50-52)$, exposure of the infant via this route at the time of PKU testing (i.e. the second postnatal day) would be likely be limited, inasmuch as effective breast-feeding is not normally established by this time (53). Although we did not measure drug levels in colostrum at the time of the PKU testing, breast milk fat content increases as lactation becomes established so that lipid-soluble drugs, such as SSRIs would transfer more readily into mature milk than into colostrum (54). Moreover, even when effective breast-feeding is established, the drug levels in breast milk are insufficient to result in the plasma concentrations observed in the infants at the time of PKU testing (48, 50-52). Pharmacologic effects may have also been secondarily expressed as altered neonatal behaviors (jitteriness, tachypnea, hypothermia, poor tone, lethargy) reported in other cohorts of exposed infants (27-30), which as behavioral states can by themselves also influence pain reactivity (32). None of these behavioral disturbances were reported among exposed infants in our cohort in the newborn period or at the time to the PKU test. Although clonazepam levels have been detected in serum of infants breast-feeding of mothers taking this medication during and after pregnancy, adverse behavioral effects have not been reported with this exposure (55).

The second mechanism that could explain our findings is altered brain development due to prolonged in utero exposure to SSRIs. Because of the dual roles of GABA agonists and 5HT in pain inhibition and as trophic developmental signals, an increase in their levels in the prenatal period may alter the receptor numbers and/or function of their receptors or postreceptor mechanisms, thereby altering postnatal behavior. Evidence from both from animal and human studies suggests that exposure to substances that increase the presence, enhance, or block the action of neurochemicals during crucial periods of fetal brain growth may alter brain structure and function, and subsequent behavior $(3,56)$. Therefore, examining the influence of prenatal medication exposure in human infants offers opportunities to investigate how levels of neurochemicals (i.e. central $5 \mathrm{HT}$ and GABAa), altered by the pharmacologic action of specific medications influences the neural ontogeny and the subsequent development of particular behaviors (regulation of pain response) mediated by those neurotransmitters. Because pain reactivity is a 5HT- and GABA-mediated process at multiple levels of the neuraxis, it is conceivable that infant pain reaction may be an ideally suited probe to examine CNS development and, in particular, the behavioral teratogenic consequences of prenatal SSRI exposure. In the context of our study, given the inhibitory role these neurotransmitters play in the pain system, the observed blunted pain reactivity may reflect an increased or altered capacity to modulate pain signals.

In animal models, prenatal exposure to SSRI medication produces a variety of adverse neuroanatomic and behavioral 


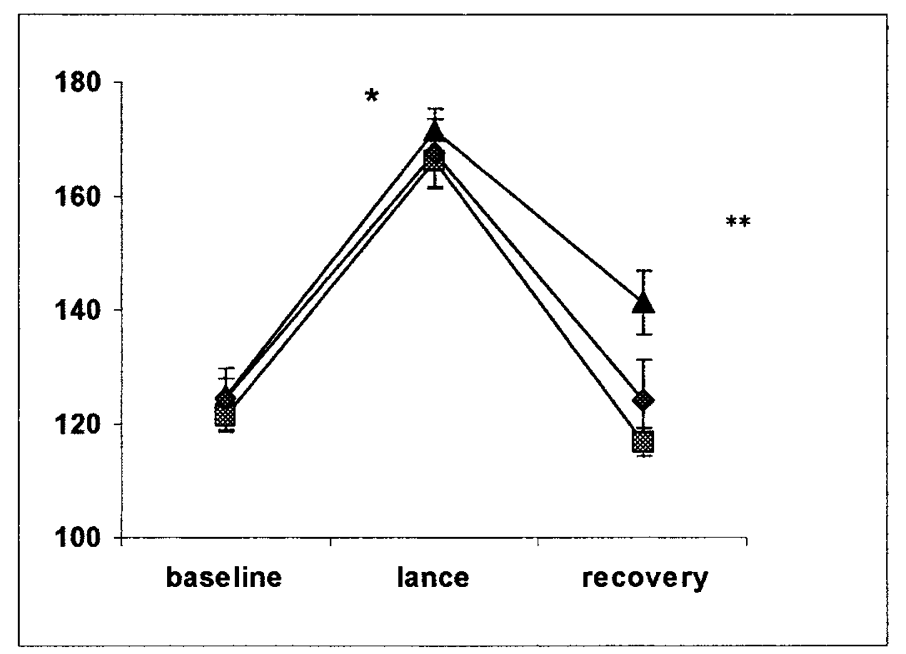

Figure 2. Hear rate response to lance from baseline (mean, bpm \pm SEM). Square, SSRI; triangle, control infants; diamond, SSRI $+.{ }^{*} p<0.05$ for repeated measures ANOVA across time epochs; ${ }^{* *} p<0.05$ between exposure groups and control infants during recovery.

LFP

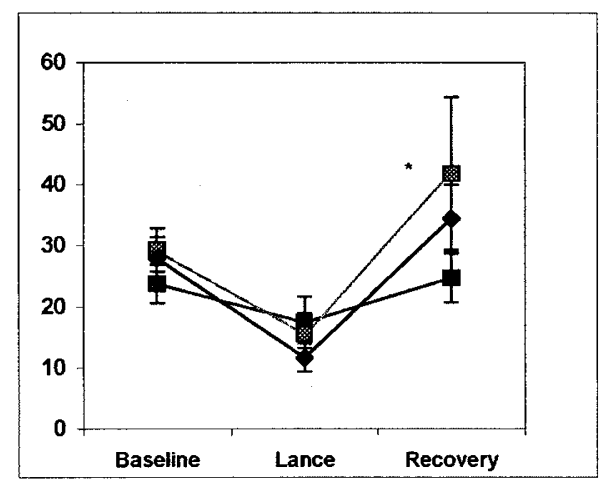

HFP

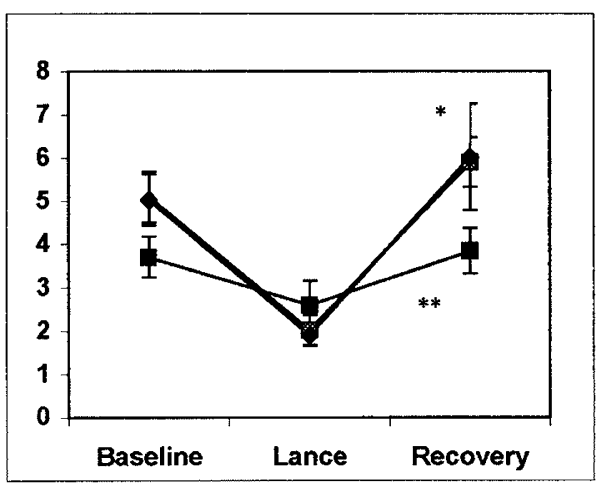

\section{LFP/HFP ratio}

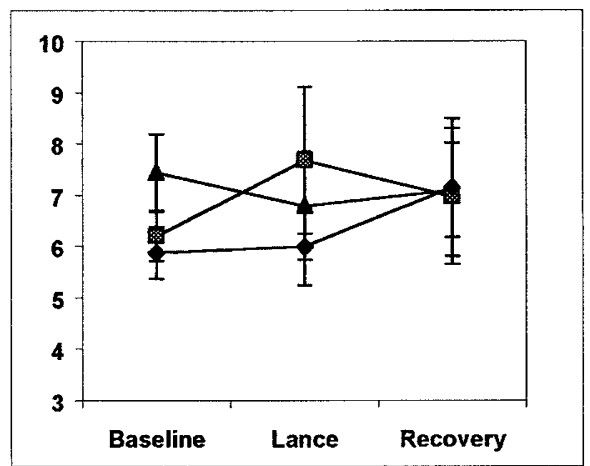

Figure 3. LFP, HFP, and LFP/HFP ratio response to heel lance from baseline (bpm/ $\mathrm{L}^{2} / \mathrm{Hz} \pm \mathrm{SEM}$ ). Square, SSRI; triangle, control infants; diamond, SSRI + . ${ }^{*} p<0.05$ for repeated measures ANOVA across time epochs; ${ }^{* *} p<0.05$ between exposure groups and control infants during recovery.

consequences, though outcomes are not consistent (15) and the long-term functional consequences are not clear. Prenatal exposure to SSRIs in rats results in a reduced number of $\beta$-adrenergic and serotonin receptors (16), abnormalities in brain serotonin receptor binding and density in the postnatal period in rats (17), and decreased numbered of 5HT receptors and altered function that persists in the mature animal (18). In an analogous experiment, Huether (4) raised the dietary content of the serotonin precursor, tryptophan in rats during pregnancy and lactation. Feeding rats this enriched diet caused a substantial retardation in the maturation of the 5HT system in the brain of rat offspring. This appears to be associated with structural abnormalities such as delayed outgrowth of 5HT axons and reduced collateral sprouting and synapse formation in the brain 
of developing rats. It has also been demonstrated that prenatal fluoxetine exposure leads to age-dependent (prepubescent) and CNS site-specific (hypothalamus, limbic system, and amygdala) alterations to the density of 5HT transporters (57) and reduced 5HT content (58). Studies of behavior in rats with postnatal serotonin agonist exposure have shown accelerated eye-opening and increased anxiety (59). Interestingly, these effects were age-dependent, appearing in early in neonatal life (15). The functional implications of these findings remain to be elucidated.

At present, there are no published studies of pain reactivity in individuals after prenatal SSRI or GABA agonist exposure, however, analogous work with cocaine-exposed infants might illustrate parallel effects. Like SSRIs, cocaine inhibits reuptake of monoamines such as $5 \mathrm{HT}(56,60)$ and as such can be considered "a pharmacologic analogue" of SSRIs. Prenatal exposure leads to increased 5HT levels in the synaptic cleft (56), resulting in up-regulation of postsynaptic receptors, delayed maturation of the serotonergic system, interference with neural migration in areas of the cortex in regions regulated by the monoaminergic system (61), and supersensitivity to catecholamines [see Mayes for review (62)], all areas that potentially adversely affect pain/stress reactivity. Emerging evidence suggests that prenatal cocaine exposure leads to an altered neurotransmitter system, activity, or receptor formation for all three monoaminergic systems (62). In a single study of acute pain responses in cocaine-exposed infants, Roumell et al. (63) reported fewer discrete negative expressions of distress and anger and more sadness at 18 mo of age. Although this study did not control for maternal-infant interaction or the effects of the care-giving environment, and therefore may not reflect the direct effects of drug exposure per se, it might provide indirect evidence that altered prenatal 5HT levels are associated with

Total respiratory power and response to lance $\left(\mathrm{L}^{2} / \mathrm{Hz} / \mathrm{m}^{2} \pm\right.$ sem).

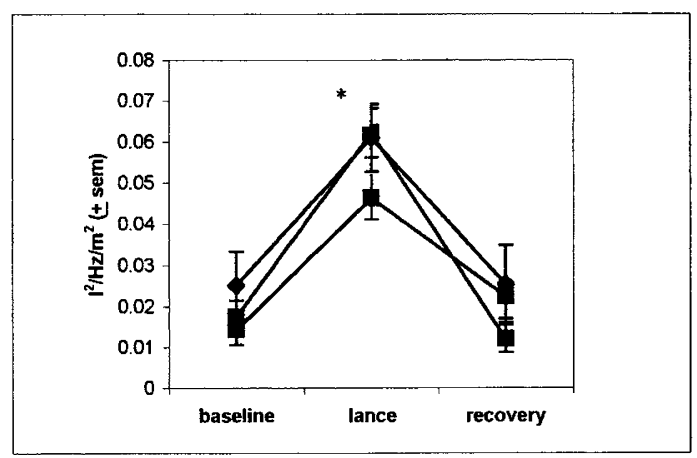

Figure 4. Total respiratory power response to heel lance from baseline $\left(\mathrm{L}^{2} / \mathrm{Hz} / \mathrm{m}^{2} \pm \mathrm{SEM}\right)$. Square, SSRI; triangle, control infants; diamond, SSRI+. $* p<0.05$ for repeated measures ANOVA across time epochs.

subsequently altered pain reactivity. Our study of pain responses in the newborn period was an attempt to examine pain reactivity (and by extension CNS development) without the confounding influence of environment and maternal care taking.
In the current study, both $\mathrm{SE}$ and $\mathrm{SE}+$ groups had similar response to the noxious event, and therefore it is not possible on the basis of this study to determine the specific effects of GABA agonists have on the developing brain. However, it is conceivable that both these medications may alter pain system function. Early exposure to BZs in rats appears to alter nervous system development by decreasing GABAa receptor function $(9,64)$ and density $(65)$, as well as the trophic action of GABA $(65,66)$. In prenatally exposed offspring in animal models, signs of abnormal behavior such as chronic anxiety, the inability to habituate to a novel environment, impaired complex discrimination $(66,67)$, impaired learning and memory $(68)$, and delayed suckling function have been observed $(69,70)$. Furthermore, prenatal exposure to diazepam and other BZs appear to alter corticotropin and noradrenergic neuron function involved with stress response systems $(9,71)$. Evidence of adverse neurobehavioral effects after prenatal BZ exposure in humans is limited and no studies of alerted pain reactivity following prenatal exposure have been reported. The effects of prenatal exposure have been limited to a description of a "floppy infant syndrome" $(72,73)$ or "withdrawal" symptoms such as hypotonia, hypothermia, respiratory depression, and poor suckling in the newborn period (74-77). Given these animal data and the role GABA may play in the developing nervous system, it is conceivable human infants' early pain behavior may also be adversely affected following prenatal exogenous GABA agonist exposure. This will have to be determined in future studies of prenatal GABA agonist exposure and pain reactivity in newborn infants.

Infant pain reactivity is directly and interactively related to emerging functional neuroanatomy, neurochemicals that regulate levels of arousal (behavioral states) and emotion during fetal development and infancy $(78,79)$, and therefore factors that disrupt pain response may be reflective of alterations to other behaviors resurfacing in some new expression, as cognitive demands change with age. Because monoaminergic-rich regions of the brain (i.e. striatum, locus ceruleus, and posterior parietal cortex) are involved with regulation of arousal and attention, exposure to SSRIs in utero may adversely affect those developing regions of the brain, similar to the effects following prenatal cocaine exposure (see Mayes for review (62)]. Altered prenatal 5HT and GABA levels may also adversely influence the infant's capacity to regulate arousal and stress rather than directly influence nociception per se. Attention and its relation to pain reactivity in SE infants remains to be studied.

Limitations. The human models needed to explain the effects of SE are complex and involve multiple neurologic, developmental, maternal genetic, and pre- and postnatal environmental factors. Not all of these factors can be controlled for in a single study, or mechanisms of action understood with available analytic models. In this preliminary study, we were not able to distinguish the effects of SSRIs from maternal stress and anxiety because we did not study a nonpharmacologically treated but depressed group of mothers, and therefore we could not account for the effects of prenatal stress alone on the developing fetus. Further, we were not able to control for unforeseen changes in levels of maternal depression/stress (in 

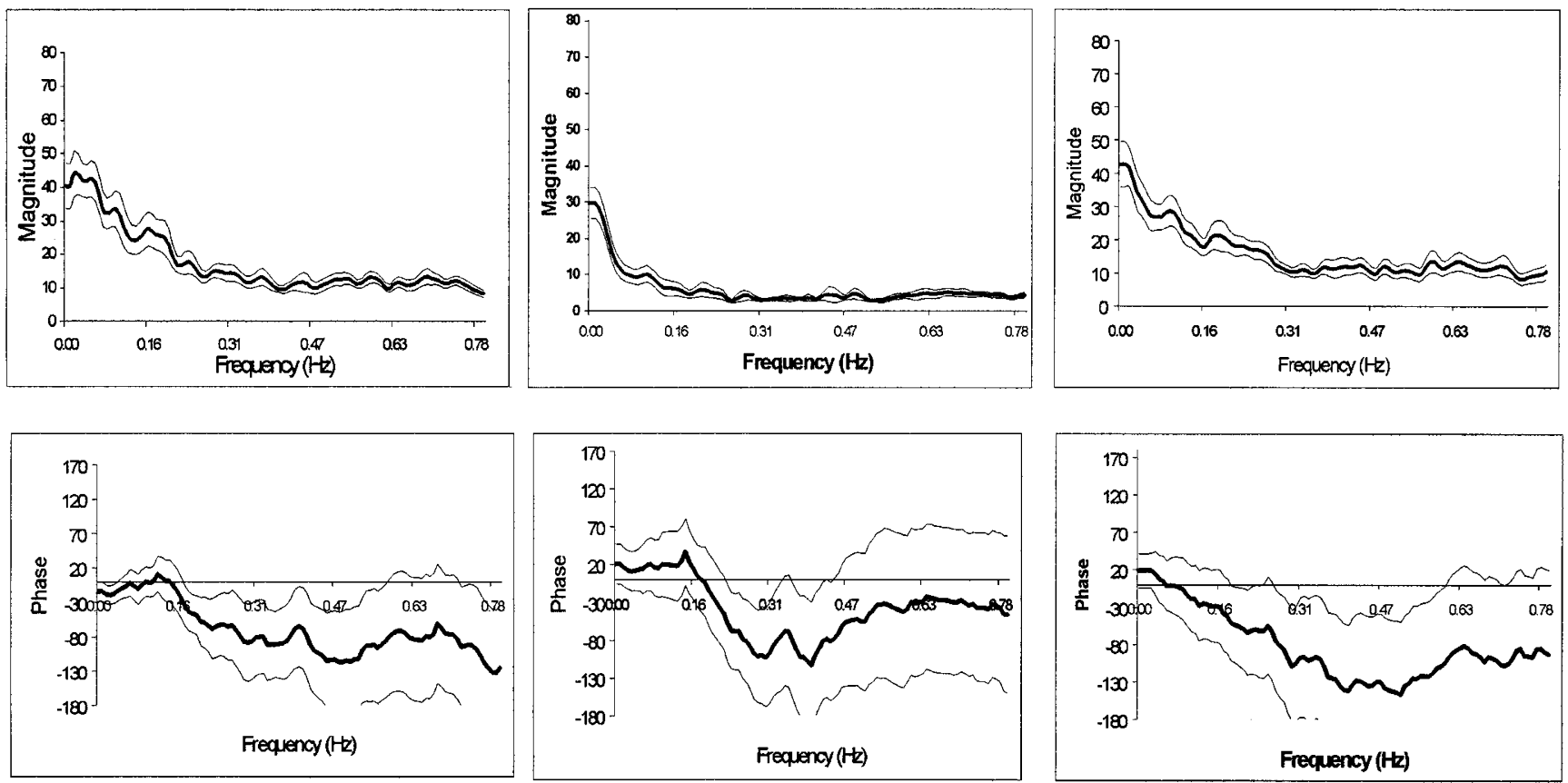

B

Baseline
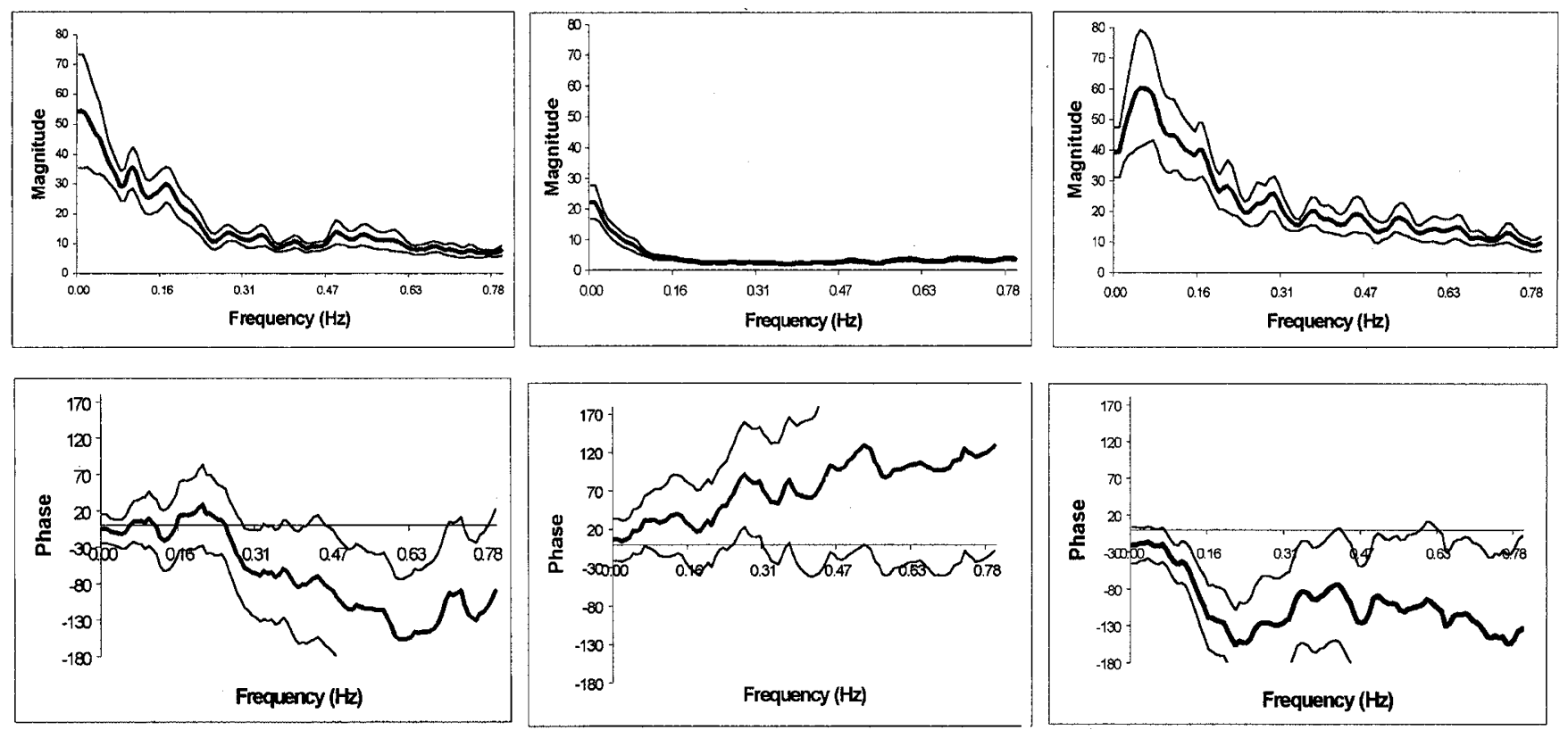

Figure 5. The complex transfer function, or frequency response, between respiratory activity and HR quantified using the cross-spectral method to yield magnitude (gain) and phase components. (A) Mean transfer function gain and phase in control infants (mean $\pm \mathrm{SEM})$. Axis for magnitude is bpm/L $/ \mathrm{m}^{2}( \pm \mathrm{SEM})$. (B) Mean transfer function gain and phase in SE infants (mean \pm SEM). Axis for magnitude is $\mathrm{bpm} / \mathrm{L} / \mathrm{m}^{2}$ ( \pm SEM). 
both directions) or medication use (i.e. benzodiazepines, switches to other medications, etc.) during the course of the study. The developmental significance of a reduced or blunted pain response remains unclear. Mechanisms that account for how SE could influence pain, and arousal and emotional development in infants need to be examined in long-term studies. This will need to include investigation of a complex model of the interaction between multiple maternal, pharmacologic environmental, and biologic variables.

In summary, this study is the first to show that antenatal exposure to SSRIs is associated with diminished facial and heart rate responses to the painful heel lance procedure at the time of PKU testing $2 \mathrm{~d}$ after birth. These altered responses could have resulted from the continued presence of the drugs that the infants received via placental transfer before birth, or from altered brain development due to prolonged in utero exposure to the SSRIs. They may also reflect the poor neonatal adaptation that has been reported in infants exposed to the drugs in the third trimester. The coadministration of BZs in $41 \%$ of the depressed women may have contributed to the altered responses. Further studies involving larger numbers of pregnant women taking SSRIs with and without BZs and whose infants are studied beyond the postnatal period are required to extend our findings. Our findings should not prejudice the clinical urgency of treating maternal depression during and after pregnancy, inasmuch as the risks of untreated or undertreated maternal depression to mothers and their offspring during and after pregnancy currently outweigh any known adverse effects of the SSRIs. Substantial work is needed to further our understanding of the effects of prenatal psychotropic medication exposure on infant development.

Acknowledgments. The authors thank Sandy Pitfield, MSc, $\mathrm{MD}$, who assisted in editing and analysis of the heart rate signals, for his invaluable contribution.

\section{REFERENCES}

1. Altshuler LL, Cohen L, Szuba MP, Burt VK, Gitlin M, Mintz J 1996 Pharmacolgic management of psychiatric illness during pregnancy: dilemmas and guidelines. Am J Psychiatry 153:529-606

2. Whitaker-Azmitia PM, Druse M, Walker P, Lauder JM 1996 Serotonin as a developmental signal. Behav Brain Res 73:19-29

3. Lauer JA, Adams PM, Johnson KM 1987 Perinatal diazepam exposure: behavioral and neurochemical consequences. Neurotoxicol Teratol 9:213-219

4. Huether G, Thomke F, Adler L 1992 Administration of tryptophan-enriched diets to pregnant rats retards the development of the serotonergic system in their offspring. Brain Res Dev Brain Res 68:175-181

5. Lauder JM 1993 Neurotransmitters as growth regulatory signals: role of receptors and second messengers. Trends Neurosci 16:233-240

6. De Pasqual E, Monteau R, Hilaire G 1994 Endogenous serotonin modulates the fetal respiratory rhythm: an in vitro study in the rat. Dev Brain Res 80:222-232

7. Smith D, Gallager D 1987 GABA, benzodiazepine and serotonergic receptor development in the dorsal raphe nucleus: electrophysiological studies. Dev Brain Res 35:191-198

8. Lauder JM, Han VK, Henderson P, Verdoorn T, Towle AC 1986 Prenatal ontogeny of the GABAergic system in the rat brain. Neuroscience 19:36-38

9. Kellogg CK 1995 Perinatal benzodiazepine modulation of GABAA receptor function influence on adaptive responses. NIDA Res Monogr 158:202-226

10. Lauder JM 1995 Ontogeny of neurotransmitter systems: substrates for developmental disabilities? Ment Retard Dev Disabil Res Rev 1:151-168

11. Fitzgerald M 1995 Pain in infancy: some unanswered questions. Pain Rev 2:77-91

12. Fitzgerald M 1997 Neonatal pharmacology of pain. In: Dickenson AH, Besson J-MR, Appleton I (eds) The Pharmacology of Pain. Springer, New York

13. Waagepetersen HS, Sonnewald U, Schousboe A 1999 The GABA paradox: multiple roles as metabolite, neurotransmitter, and neurodifferentiative agent. J Neurochem $73: 1335-1342$
14. Gaiarsa JL, McLean H, Congar P, Leinekugel X, Khazipov R, Tseeb V, Ben-Ari Y 1995 Postnatal maturation of gamma-aminobutyric acid A and B-mediated inhibition in the CA3 hippocampal region of the rat. J Neurobiol 26:339-349

15. Vorhees CV, Acuff-Smith KD, Schilling MA, Fisher JE, Moran MS, Buelke-Sam J 1994 A developmental neurotoxicity evaluation of the effects of prenatal exposure to fluoxetine in rats. Fundam Appl Toxicol 23:194-205

16. De Ceballos ML, Benedi A, Urdin C, Del Rio J 1985 Prenatal exposure of rats to antidepressant drugs down-regulates beta-adrenoceptors and 5-HT2 receptors in cerebral cortex: lack of correlation between 5-HT2 receptors and serotonin-mediated behaviour. Neuropharmacology 24:947-952

17. Montero D, De Ceballos ML, Del Rio J 1990 Down-regulation of 3H-imipramine binding sites in rat cerebral cortex after prenatal exposure to antidepressants. Life Sci 46:1619-1626

18. Cabrera TM, Battaglia G 1994 Delayed Decreases in brain 5-hydroxytryptamine $2 \mathrm{~A} / 2 \mathrm{C}$ receptor density and function in male rat progeny following prenatal fluoxetine. J Pharma Experi Therapeutics 269:637-645

19. Pastuszak A, Schick-Boschetto B, Zuber C, Feldkamp M, Pinelli M, Sihn S, Donnenfeld A, McCormack M, Leen-Mitchell M, Woodland C, et al. 1993 Pregnancy outcome following first-trimester exposure to fluoxetine (Prozac). JAMA 269:22462248

20. Goldstein DJ 1991 Fluoxetine-exposed pregnancies. Clin Res 39:768A

21. Vendittelli F, Alain J, Nouaille Y, Brosset A, Tabaste JL 1995 A case of lipomeningocele with fluoxetine (and alprazolam, vitamins B1 and B6, heptaminol) prescribed during pregnancy. Eur J Obstet Gynecol 58:85-86

22. Stanford MS, Patton JH 1992 In utero exposure to fluoxetine HCI increases hematoma frequency at birth. Pharmacol Biochem 45:959-962

23. Nulman I, Rovet J, Stewart DE, Wolpin J, Gardner HA, Theis JG, Kulin N, Koren G 1997 Neurodevelopment of children exposed in utero to antidepressant drugs. N Engl J Med 336:258-262

24. Koren G, Nulman I, Addis A 1998 Outcome of children exposed in utero to fluoxetine: a critical review. Depress Anxiety 8(suppl 1):27-31

25. Kulin NA, Pastuszak A, Koren G 1998 Are the new SSRIs safe for pregnant women? Can Fam Physician 44:2081-2083

26. Spencer MJ 1993 Fluoxetine hydrochloride (Prozac) toxicity in a neonate. Pediatrics 92:721-722

27. Lester BM, Cucca J, Andreozzi L, Flanagan P, Oh W 1993 Possible association between fluoxetine hydrochloride and colic in an infant. J Am Acad Child Adolesc Psychiatry 32:1253-1255

28. Nordeng H, Lindemann R, Perminov KV, Reikvam A 2001 Neonatal withdrawal syndrome after in utero exposure to selective serotonin reuptake inhibitors. Acta Paediatr 90:288-291

29. Cohen LS, Heller VL, Bailey JW, Grush L, Ablon JS, Bouffard SM 2000 Birth outcomes following prenatal exposure to fluoxetine. Biol Psychiatry 48:996-1000

30. Chambers CD, Johnson KA, Dick LM, Felix RJ, Jones KL 1996 Birth outcomes in pregnant women taking fluoxetine. N Engl J Med 335:1010-1015

31. Prechtl HF 1974 The behavioral states of the newborn infant (a review). Brain Res 76:185-212

32. Grunau RE, Craig KD 1987 Pain expression in neonates: facial action and cry. Pain 28:395-410

33. Grunau RVE, Johnston CC, Craig KD 1990 Neonatal facial and cry responses to invasive and non-invasive procedures. Pain 42:295-305

34. Craig KD, Whitfield MF, Grunau RV, Linton J, Hadjistavropoulos HD 1993 Pain in the preterm neonate: behavioural and physiological indices. Pain 52:287-299

35. Grunau RVE, Oberlander TF, Holsti L, Whitfield MF 1998 Bedside application of the Neonatal Facial Coding System in pain assessment of premature neonates. Pain 76:277-286

36. Lilley CM, Craig KD, Grunau RE 1997 The expression of pain in infants and toddlers: developmental changes in facial action. Pain 72:161-170

37. Saul JP, Berger RD, Albrecht P, Stein SP, Chen MH, Cohen RJ 1991 Transfer function analysis of the circulation: unique insights into cardiovascular regulation. Am J Physiol 261:H1231-H1245

38. Fox NA, Porges SW 1985 The relation between neonatal heart period patterns and developmental outcome. Child Dev 59:28-37

39. Porter FL, Porges SW, Marshall RE 1988 Newborn pain cries and vagal tone: parallel changes in response to circumcision. Child Dev 59:495-505

40. Litvack DA, Oberlander TF, Carney LH, Saul JP 1995 Time and frequency domain methods for heart rate variability analysis: a methodological comparison. Psychophysiology 32:492-504

41. Oberlander TF, Berde CB, Lam KH, Rappaport LA, Saul JP 1995 Infants tolerate spinal anesthesia with minimal overall autonomic changes: transfer function analysis of respiratory sinus arrhythmia in former premature infants undergoing hernia repair. Anesth Analg 80:20-27

42. Oberlander TF, Berde CB, Lam KH, Saul JP 1996 Halothane causes withdrawal of cardiac sympathetic modulation in infants: transfer function analysis of respiratory sinus arrhythmia. Pediatr Res 40:710-717

43. Hanna BD, Saul JP, Cohen RJ, Stark AR 1990 Transfer function analysis of respiratory sinus arrhythmia: developmental changes in sleeping premature infants. Circulation 82(suppl III):334(abstr)

44. Oberlander TF, Grunau RE, Whitfield MF, Fitzgerald C, Pitfield S, Saul JP 2000 Biobehavioral pain responses in former extremely low birth weight infants at four months' corrected age. Pediatrics 105:e6

45. Brouillette RT, Morrow AS, Weese-Mayer DE, Hunt CE 1987 Comparison of respiratory inductive plethysmography and thoracic impedance for apnea monitoring. J Pediatr 111:377-383

46. Berger RD, Akselrod S, Gordon D, Cohen RJ 1986 An efficient algorithm for spectral analysis of heart rate variability. IEEE Trans Biomed Eng 33:900-904 
47. Berger RD, Saul JP, Cohen RJ 1989 Transfer function analysis of autonomic regulation. I. canine atrial rate response. Am J Physiol 256:H142-H152

48. Kim J, Misri S, Kent N, Oberlander T, Rurak DW, Riggs KW 1999 Comparison of fetal and neonatal fluoxetine and paroxetine exposure during the perinatal period in humans. AAPS PharmSci 1:S96

49. Hiemke C, Härtter S 2000 Pharmacokinetics of selective serotonin reuptake inhibitors. Pharmacol Ther 85:11-28

50. Öhman R, Hägg S, Carleborg L, Spigset O 1999 Excretion of paroxetine into breast milk. J Clin Psychiatry 60:519-523

51. Stowe ZN, Owens MJ, Landry JC, Kilts CD, Ely T, Llewellyn A, Nemeroff CB 1998 Sertraline and desmethylsertraline in human breast milk and nursing infants. Am J Psychiatry 155:1643-1644

52. Tuddin A, Ito S, Koren G 1996 Excretion of fluoxetine and its metabolite, norfluoxetine, in human breast milk. J Clin Pharmacol 36:42-47

53. Neville MC 1999 Physiology of lactation. Clin Perinatol 26:251-279

54. Jenness R 1979 The composition of human milk. Semin Perinatol 3:225-239

55. Birnbaum CS, Cohen LS, Bailey JW, Grush LR, Robertson LM, Stowe ZN 1999 Serum concentrations of antidepressants and benzodiazepines in nursing infants: a case series. Pediatrics 104:e11

56. Mayes LC, Grillon C, Granger R, Schottenfeld R 1998 Regulation of arousal and attention in preschool children exposed to cocaine prenatally. Ann N Y Acad Sci 846:126-143

57. Cabrea-Vera TM, Battaglia G 1998 Prenatal exposure to fluoxetine (Prozac) produces site-specific and age-dependent alterations in brain serotonin transporters in rat progeny: evidence from autoradiographic studies. J Pharm Exp Therapeutics 286:1474-1481

58. Cabrea-Vera TM, Garcia F, Battaglia G 1997 Effect of prenatal fluoxetine (Prozac) exposure on brain serotonin in prepubescent and adult male rat offspring. J Pharm Exp Therapeutics 280:138-45

59. Borella A, Bindra M, Whitaker-Azmitia PM 1997 Role of the 5-HT1A receptor in development of the neonatal rat brain: preliminary behavioral studies. Neuropharmacology 36:445-450

60. Whitaker-Azmitia PM 1998 Role of the neurotropic properties of serotonin in the delay of brain maturation induced by cocaine. Ann N Y Acad Sci 846:158-164

61. Akbari HM, Kramer HK, Whitaker-Azmitia PM, Spear LP, Azmitia EC 1992 Prenatal cocaine exposure disrupts the development of the serotonergic system. Brain Res $572: 57-63$

62. Mayes LC 1999 Developing brain and in utero cocaine exposure: effects on neural ontogeny. Dev Psychopathol 11:685-714

63. Roumell N, Wille D, Abramson L, Delaney V 1997 Facial expressivity to acute pain in cocaine exposed toddlers. Infant Mental Health Journal 18:274-281
64. Chesley S, Lumpkin M, Schatzki A, Galpern WR, Greenblatt DJ, Shader RI, Miller LG 1991 Prenatal exposure to benzodiazepine-I. Prenatal exposure to lorazepam in mice alters open-field activity and GABAA receptor function. Neuropharmacology. 30:53-58

65. Livezey GT, Marczynski TJ, Isaac L 1986 Enduring effects of prenatal diazepam on the behavior, EEG, and brain receptors of the adult cat progeny. Neurotoxicology $7: 319-333$

66. Livezey GT, Radulovacki M, Isaac L, Marczynski TJ 1985 Prenatal exposure to diazepam results in enduring reductions in brain receptors and deep slow wave sleep. Brain Res 334:361-365

67. Pankaj V, Brain PF 1991 Effects of prenatal exposure to benzodiazepine-related drugs on early development and adult social behaviour in Swiss mice, I: agonists. Gen Pharmacol 22:33-41

68. Gai N, Grimm VE 1982 The effect of prenatal exposure to diazepam on aspects of postnatal development and behavior in rats. Psychopharmacology 78:225-229

69. Kellogg CK 1988 Benzodiazepines: influence on the developing brain. Prog Brain Res 73:207-228

70. Schlumpf M, Parmar R, Schreiber A, Ramseier HR, Butikofer E, Abriel H, Barth M, Rhyner T, Lichtensteiger W 1992 Nervous and immune systems as targets for developmental effects of benzodiazepines. A review of recent studies. Dev Pharmacol Ther 18:145-158

71. Inglefield JR, Bitran D, Olschowka JA, Kellogg CK 1993 Selective effects on CRF neurons and catecholamine terminals in two stress-responsive regions of adult rat brain after prenatal exposure to diazepam. Brain Res Bull 31:353-359

72. Gillberg C 1977 "Floppy infant syndrome" and maternal diazepam. [letter] Lancet $2: 244$

73. Besunder JB, Reed MD, Blumer JL 1988 Principles of drug biodisposition in the neonate. A critical evaluation of the pharmacokinetic-pharmacodynamic interface. Clin Pharmacokinet 14:261-286

74. Fisher JB, Edgren BE, Mammel MC, Coleman JM 1985 Neonatal apnea associated with maternal clonazepam therapy: a case report. Obstet Gynecol 66:34S-35S

75. Mazzi E 1977 Possible neonatal diazepam withdrawal: a case report. Am J Obstet Gynecol 129:586-587

76. Rementeria JL, Bhatt K 1977 Withdrawal symptoms in neonates form intrauterine exposure to diazepam. J Pediatrics 90:123-126

77. Whitelaw AGL, Cummings AJ, McFadyen IR 1981 Effect of maternal lorazepam on the neonate. BMJ 282:1106-1108

78. Grunau RE 2000 Long-term consequences of pain in human neonates. In: Anand KJS, Stevens BJ, McGrath PJ (eds) Pain in Neonates, 2nd Ed. Elsevier Science, Amsterdam

79. Dahl RE 1996 The regulation of sleep and arousal. Dev Psychopathol 8:3-27 